\title{
Total electron yields and stopping power of protons colliding with NaCl-type insulator surfaces II
}

\author{
Andrea J. García and J. E. Miraglia \\ Instituto de Astronomía y Física del Espacio. Consejo \\ Nacional de Investigaciones Científicas y Técnicas and \\ Departamento de Física. Facultad de Ciencias \\ Exactas y Naturales. Universidad de Buenos Aires. \\ Casilla de Correo 67, Sucursal 28, \\ (C1428EGA) Buenos Aires, Argentina.
}

(Dated: August 12, 2013)

\begin{abstract}
We re-calculate electron yields and stopping power of protons colliding with surfaces of NaCl-type insulators. In this article, the projectile is considered to move taking into account the static and polarization potentials with all the individual ions forming the surface lattice unlike our previous work (Phys. Rev. A 75, 042904 (2007)) where the projectile was considered to move in an homogeneous planar potential. Substantial differences (up to forty percent of increment) have been found especially when the projectile incident angle approaches the critical one. We compare our prediction for electron yield and stopping power with the available experimental data for LiF, $\mathrm{KI}$ and $\mathrm{KCl}$.
\end{abstract}

PACS numbers: 34.50.Bw, 79.20.Rf, 71.10.Ca 


\section{INTRODUCTION}

In our previous article [1] (here refereed to as I), we reported electron yields and stopping power of proton colliding with surfaces of sixteen NaCl-type insulators formed with four alkalis $\mathrm{Li}^{+}, \mathrm{Na}^{+}, \mathrm{K}^{+}$and $\mathrm{Rb}^{+}$and four halides $\mathrm{F}^{-}, \mathrm{Cl}^{-}, \mathrm{Br}^{-}$and $\mathrm{I}^{-}$. Proton energies were considered to range between $100 \mathrm{kev}$ to $1 \mathrm{Mev}$. The (classical) movement of the projectile was calculated under planar channeling condition. In that case, the interaction with the surface is described by a potential that depends only on the distance to the surface. In this work, we consider a more realistic surface, allowing the projectile to interact with all the individual ions. In Ref. [2] (hereafter referred to as II), we called this feature punctual channeling. We decided to undertake this task -which consumes a long computing timebecause we found substantial differences as compared with the planar model, due to different trajectories.

The basic considerations we assume here are:

1i) The insulator surface is considered to be composed by an array of alkali and halide ions at the places given by the crystal parameters, and the local electronic density is described by the Hartree Fock wave functions of the isolated ions [3]. This is that we call the grid of independent ion model (GII).

2i) The trajectory of the projectile has been calculated classically, considering the interaction (static and dynamic polarization) with every single ion of the grid. The static and dynamic potentials used are tabulated in Table I of Ref. I

3i) The differential probabilities have been calculated using the Levine Louie response function [4] where the gap, in accordance with the GII model, is considered to be the ionization energy of the isolated ion, as in I. For each trajectory, the stopping power and the electron yield are calculated at each segment of the collision and integrated along all the classical projectile path.

4i) In accordance with the grid of independent ions used, electrons were counted when the energy provided by the projectile was equal or larger than the binding energy. Following the collision, we assume that there is Auger decay and so the emission of the inner layers has been multiply by two.

We report here a complete set of electron yields (total number of emitted electrons) and stopping power of protons colliding grazingly with sixteen $\mathrm{NaCl}$-type insulator surfaces built 
with the four alkalis $\mathrm{Li}^{+}, \mathrm{Na}^{+}, \mathrm{K}^{+}$and $\mathrm{Rb}^{+}$and the four halides $\mathrm{F}^{-}, \mathrm{Cl}^{-}, \mathrm{Br}^{-}$and $\mathrm{I}^{-}$. As in Ref. II, we use a Runge Kutta code to describe the projectile motion. Thirty two projectile nearest neighbors were considered, i.e. $4 \times 4 \times 2$. Hundreds of random couple coordinates were considered to average the incident projectile initial starting point with respect to the grid. For a given velocity, we have considered 13 incident polar angles $\theta_{i}$. For each polar angle $\theta_{i}$, we considered 800 equally-spaced azimuth angles $\left(\varphi_{i}\right)$ between 0 and 45 degrees with respect to the index $[1,0,0]$. For each value of $\theta_{i}$ and $\varphi_{i}$, around $10^{4}$ random trajectories were required for the calculation to converge, and it took several hours of CPU computing time. Step-to-step, we add the stopping and yield produced in the interval using the same differential probabilities as in I. The present punctual channeling calculation takes about two to three orders of magnitude more of CPU computing time than the planar channeling due to the more complicated projectile trajectories that arise. We think that this effort is justified because far more physical details are included which are decisive as the projectile approaches the surface with inclination approaching the critical angle of penetration.

In Figs. 1 and 2, we plot the total electron production for the sixteen insulator surfaces as a function of the proton penetration angle normalized to the critical one (See Table II of Ref. I). We consider all possible projectiles trajectories including the ones that penetrate and remains in the bulk. In Figs. 3 and 4 we plot the total energy loss by the proton. When compared with similar calculations using the planar model (see Figs 5-8 of Ref. I), the following can be stated:

i) At small incident angles both models seem to produce roughly the same predictions (although if we look carefully, punctual channeling is always larger).

ii) As we increase the incident angle, the punctual model produces many more yields and stopping than the planar. At the critical angle $\left(\theta \approx \theta_{c}\right)$ the punctual model produces between 37 and $40 \%$ (KI case) for the total yields and between 23 and $31 \%$ (for KI) for the total stopping.

Next we proceeded to compare with the available experiments. At this stage it is important to note that, when we compare our results with the experimental values:

a) We have accounted for coincidence. That is, we consider the electrons emitted by protons whose trajectories penetrated and then left the insulator or simply rebounded, regardless of the outgoing angle. The ones that go below the second layer are not considered.

b) We considered that half the electrons emitted do not leave the insulator because they 
are lost to the bulk and not counted by the experiment.

c) In the theoretical computing we did not account for secondary electron emissions, i.e. we did not consider cascades produced by primary electrons during the movement through the insulator.

d) The surface was considered to be perfect without terraces or any other unevenness.

In Fig. 5, the upper figure shows the energy loss as a function of the incident energy for the KI insulator when the incident angle is $0.5 \mathrm{deg}$. as a function of the projectile energy. The theoretical values are in excellent agreement with the experimental data [5]. The stopping increases with the projectile energy. In this case the critical angle for 500 kev protons is of the order of $0.5 \mathrm{deg}$., so at larger impact energies the number of outgoing projectiles decreases.

In the lower figure we plot the electron emission and the stopping as a function of the proton angle normalized to the critical angle when the proton energy is $100 \mathrm{kev}$ for the same insulator. In this case the agreement is not so good as before and we attributed it to the fact that the impact energy is not large enough yet. The experimental curve shows a soft maximum, not present in our model.

In Fig. 6 we plot the electron emission for three incident proton energies, 100, 200 and 300 kev on the LiF insulator. In this case, the agreement between theoretical and experimental values is good except for the lowest impact energy. Again this underestimation may be attributed to the fact that the model is out of range of validity. The experiments in Figs. 5 and 6 correspond to Winter and collaborators [5].

In Fig. 7 we show a comparison with another set of experiments [6, 7] for the electron yield and stopping for $\mathrm{KCl}$ and $\mathrm{LiF}$. In this case, our lower values display a substantial disagreement with the experimental data. In the case of stopping we have a difference in percentage of $23-36 \%$ for the $\mathrm{LiF}$ and between 15 and $30 \%$ for the $\mathrm{KCl}$ insulator. For the electron emission, the difference is still grater, about $70-80 \%$, for the $\mathrm{LiF}$ and $\mathrm{KCl}$, respectively. There seems to be an incompatibility between the experiments, at $300 \mathrm{kev}$ protons where the experiments account for about 60 electrons while the other one, at 500 kev account for twice as many. Our theory cannot reproduce this jump. Experiments are welcome to test the present model. 


\section{ACKNOWLEDGMENTS}

The authors acknowledge financial support from CONICET, UBACyT and ANPCyT of Argentina.

[1] A. J. García and J. E. Miraglia, Phys. Rev. A 75, 042904 (2007).

[2] A. J. García and J. E. Miraglia, Phys. Rev. A 74, 012902 (2006).

[3] E. Clementi, and C. Roetti, At. Data and Nucl. Data Tables 14, 177 (1974).

[4] Z. H. Levine, S.G. Louie, Phys. Rev. B 25, 6310 (1982).

[5] M. S. Gravielle, I. Aldazabal, A. Arnau, V. H. Ponce, J. E. Miraglia, F. Aumayr, S. Lederer and H. Winter, Phys. Rev. A 76 (2007) 012904.

[6] K. Kimura, G. Andou, K. Nakajima, Phys. Rev. Lett. 81, 5438 (1998).

[7] K. Kimura, G. Andou, K. Nakajima, Nucl. Instr. and Meth. B 164, 933 (2000). 


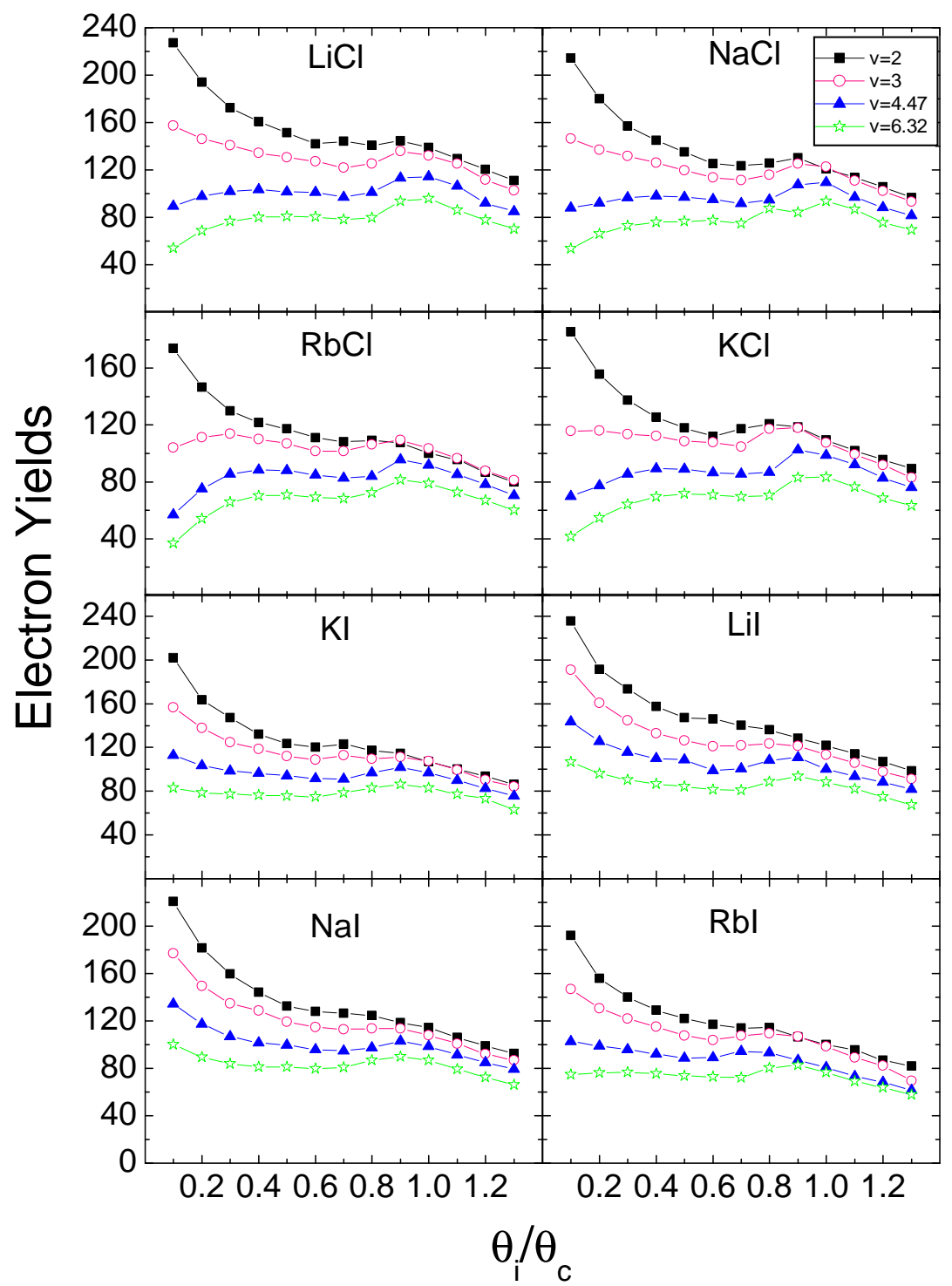

FIG. 1: Number of total electron production in all directions as a function of the proton penetration angle normalized to the critical one [1] for different velocities and insulators. The calculations consider all possible projectiles trajectories including the ones that penetrate and remain in the bulk. 


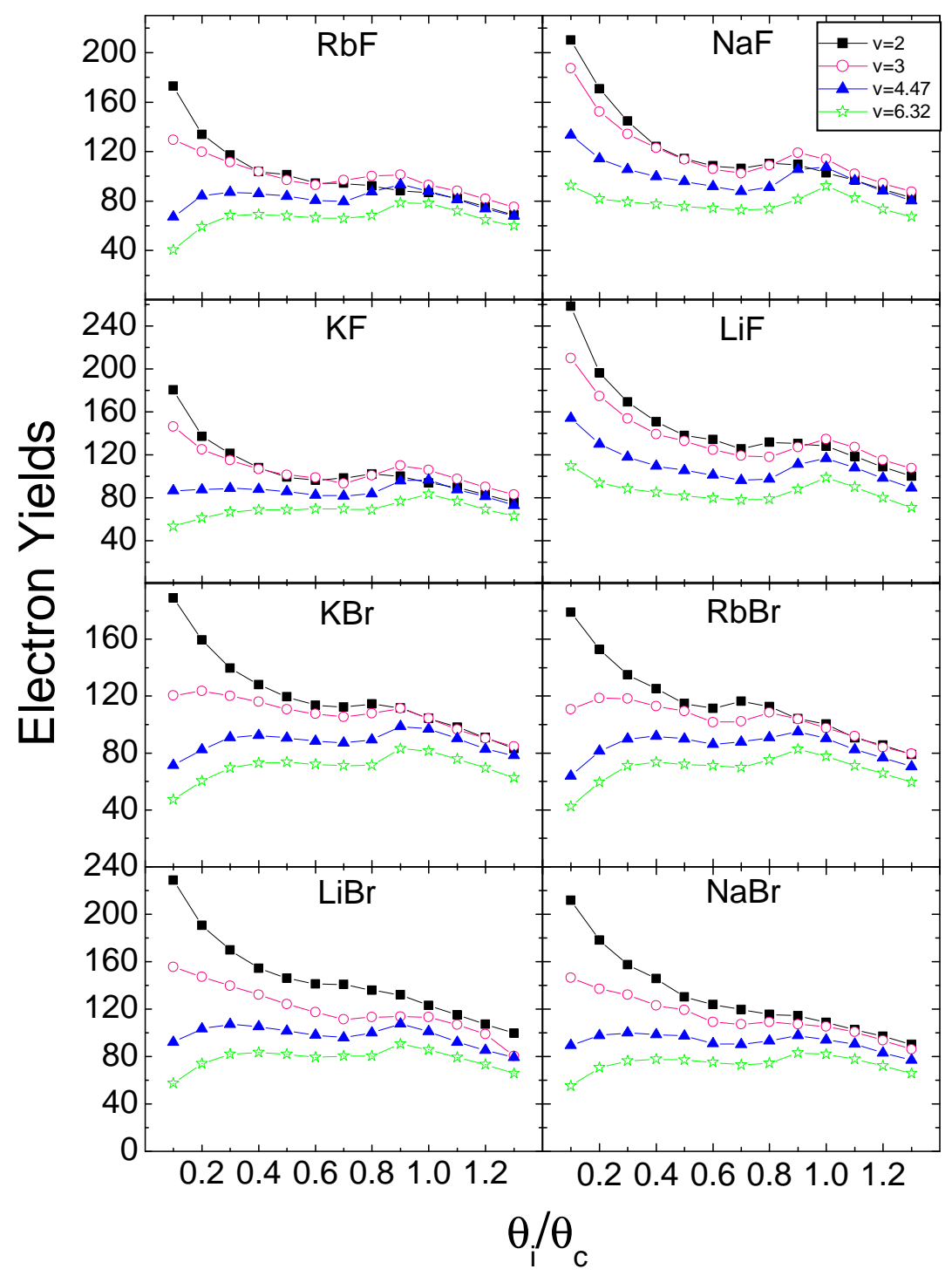

FIG. 2: Same as Fig.1 for different insulators as indicated. 


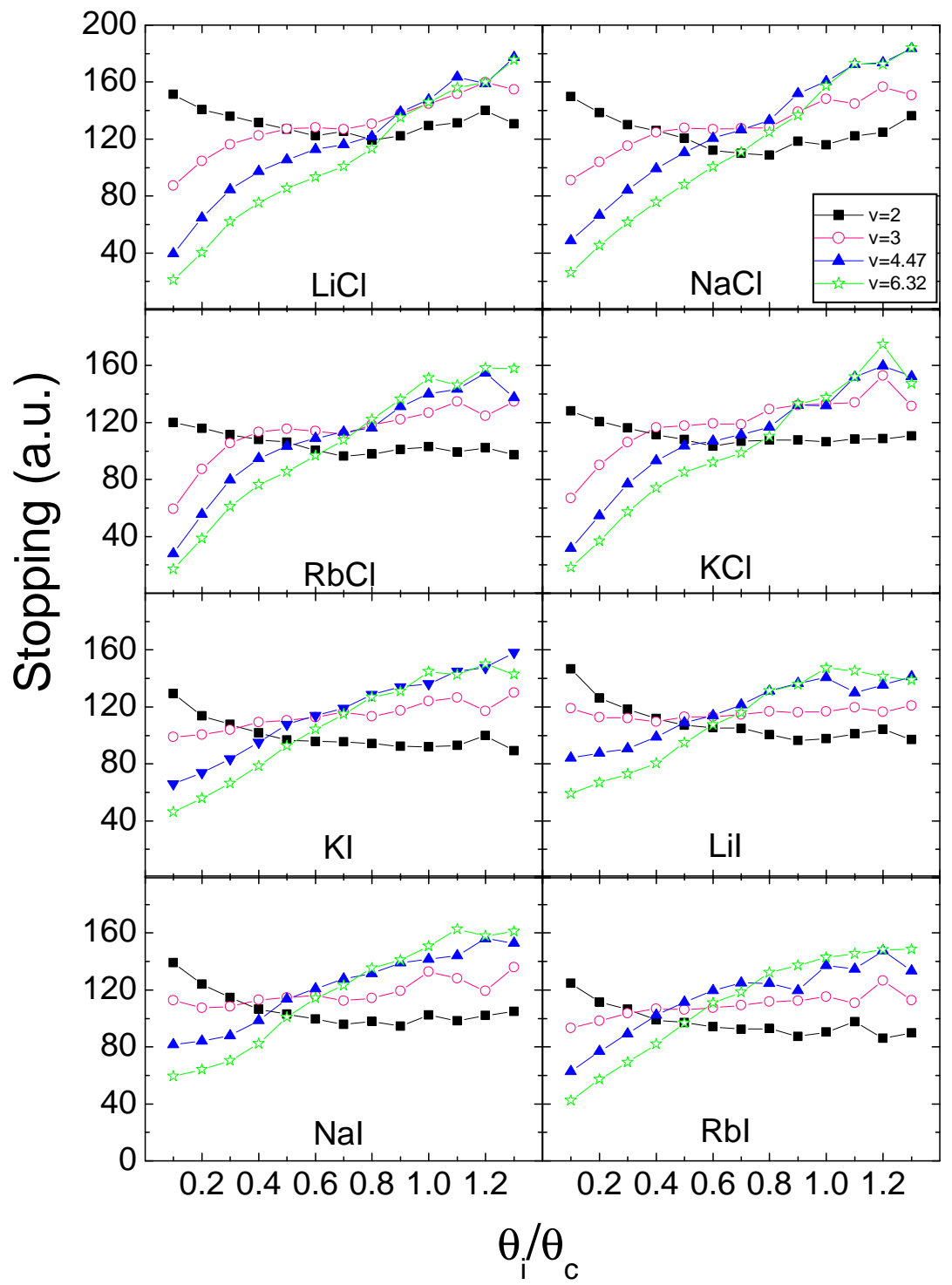

FIG. 3: Stopping power as a function of the proton penetration angle normalized to the critical one [1] for different velocities and insulators. 


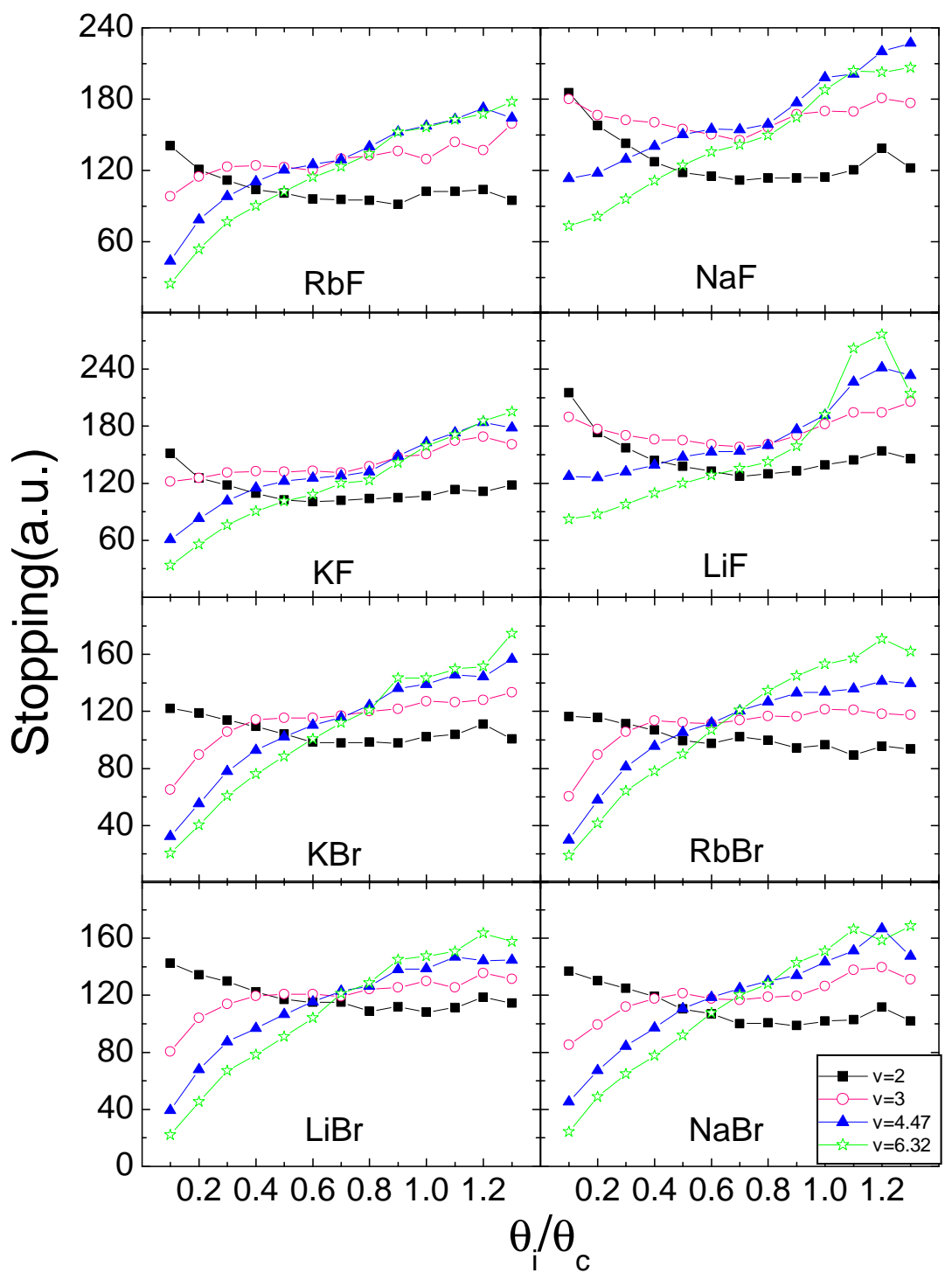

FIG. 4: Same as Fig.3, for different insulators as indicated. 

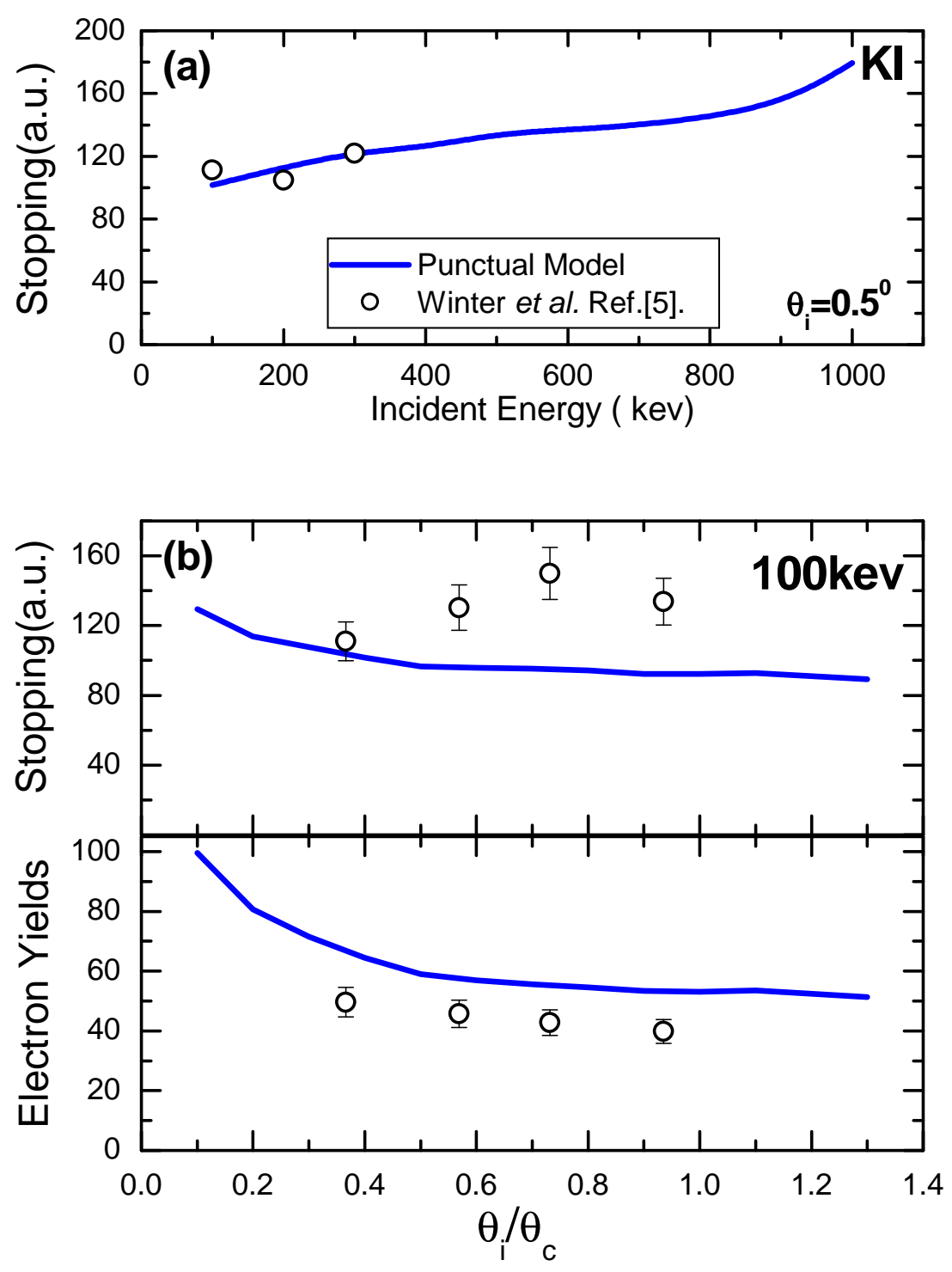

FIG. 5: (a) Stopping power as a function of the incident energy at incident angle 0.5 deg. on KI insulator [100] surface. The solid line denotes our theoretical result and the open circles the experimental values [5]. (b) Figure above: stopping power ; figure below: electron emission as a function of the proton penetration angle normalized to the critical one [1] at $100 \mathrm{kev}$ for KI insulator, calculated in coincidence when both electron and projectile end in the vacuum. The solid lines denote the theoretical results and the open circles the experimental values [5]. 


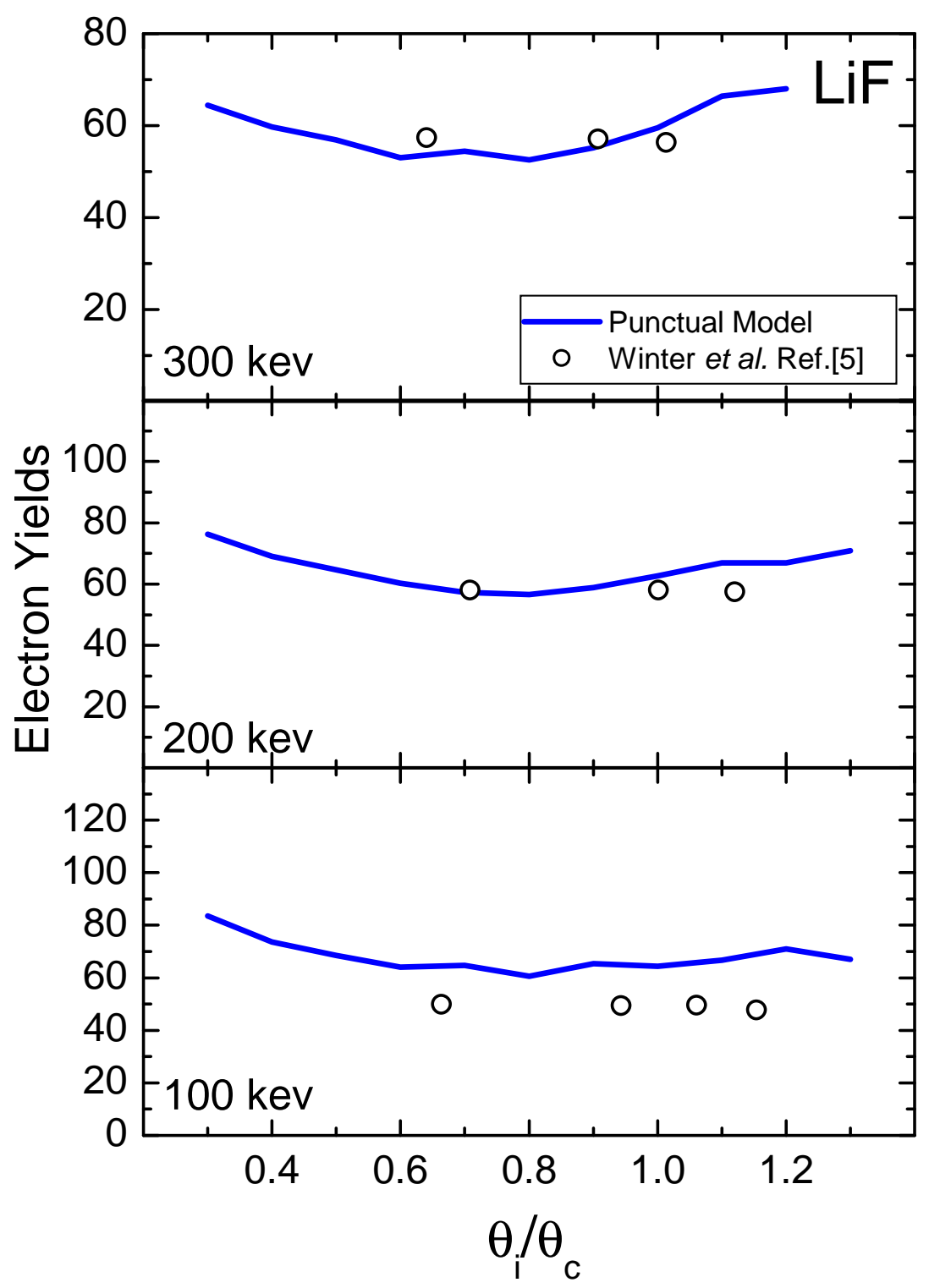

FIG. 6: Number of total electron production as a function of the proton penetration angle normalized to the critical one 1] for different impact energies on LiF insulator [100] surface, calculated in coincidence when both electron and projectile end in the vacuum. The solid lines denote the theoretical results and the open circles the experimental values [5]. 


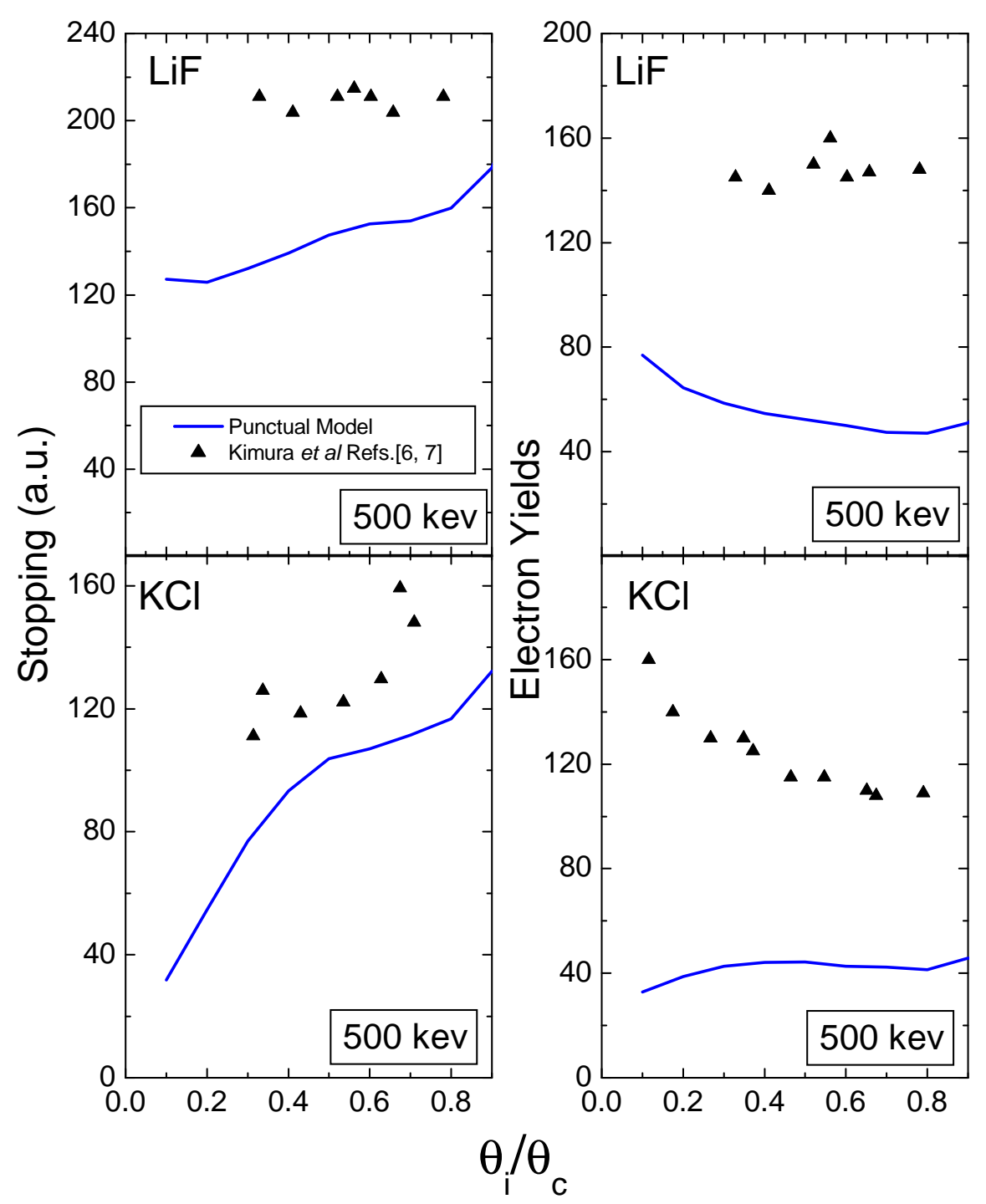

FIG. 7: Stopping power and electron yields as a function of the incident energy for $\mathrm{KCl}$ and $\mathrm{LiF}$ at 500 kev. incident energy The solid lines denote the theoretical results and the full triangles the experimental values [6, 7]. The electron yields is calculated in coincidence when both electron and projectile end in the vacuum. 